\title{
Lithopedion with seven years of evolution: Case Report
}

Warfa K. ${ }^{1}$ MBChB, Konya W.P. ${ }^{2}$ MBChB,MMed (ObGyn), 1-Resident, Department of Obstetrics and Gynaecology. Aga Khan University, Nairobi, Kenya. 2-St Mary's Hospital, Nairobi. Correspondence: Dr. Khadija Warfa, Department of Obstetrics and Gynaecology. Aga Khan University, P.0. Box 30270, Nairobi, Kenya. Email: khadija.warfa@aku.edu

\section{Summary}

Lithopedion is derived from the Greek words lithos, meaning stone, and paidion, meaning child. It describes an extrauterine fetus that has become calcified. It requires the presence of a medically undetected extrauterine pregnancy with continued asepsis of the products of conception. Earliest case of a lithopedion was described in 1582 . To date 330 cases have been reported in literature. We report the case of a seven year calcified abdominal pregnancy in a woman on follow-up for mental illness. The case brings to light the benefits of thorough history and physical examination.

\section{Case report}

A 36 year old para $1+0$ woman presented with a seven year history of lower abdominal swelling following a natural conception. She had noticed quickening at around the fifth month and started appreciating fetal movements had booked for routine antenatal care. Unfortunately, she did not have a booking or any subsequent ultrasounds done due to financial constraints. Her subsequent antenatal care was inconsistent. She however experienced loss of fetal movements but did not seek any professional advice. She was however conscious of her increasing abdominal girth and this raised curiosity among her peers and relatives.

Her past obstetric and gynecological history was not significant. She was however on follow-up for a psychiatric disorder at a local institution. She was single and still dependant on her mother. When she presented to us she was in good general condition. The abdominal examination revealed a firm mass of approximately 22 weeks gestation, associated with mild abdominal tenderness. The rest of the systemic exam was unremarkable. An abdominal Ultra sound showed an abdominal pregnancy of approximately 28 weeks gestation, with an empty uterus.

Upon obtaining an informed consent, we performed an explorative laparatomy. An extended midline incision was used; omental adhesions onto the anterior abdominal wall were encountered during abdominal entry. Following dissection, a lithopedion adherent onto the omentum, and firmly adherent to the right ovary was identified (Fig. 1). No separate placental tissue was identified. The uterus, the left ovary and fallopian tubes were normal (Fig. 2). The lithopedion was separated from the omentum, and removed with the adherent ovary (Fig. 3). Further exploration revealed normal abdominal viscera and the abdomen was closed. Postoperative follow up was uneventful. Histopathology of the lithopedion revealed a calcified fetus that weighed $1400 \mathrm{~g}$, at approximately 28 weeks gestation with external features that appeared normal.

\section{Discussion}

The diagnosis of lithopedion requires the presence of a medically undetected extrauterine pregnancy with continued asepsis of the products of conception (1). After the earliest descriptive account in 1582, only about 330 cases have been reported in literature to date (1-3). In recent times the occurrence of lithopedion has been reduced by the availability of better prenatal care and imaging techniques that locate a pregnancy, differentiating between intrauterine and extrauterine pregnancies (4).Complications caused by the presence of a lithopedion include: intestinal obstruction(1), pelvic abscess (5) tubal infertility(6) and cephalopelvic disproportion complicating a subsequent uterine pregnancy(7).

In our patient the diagnosis was made 7 years following the pregnancy. Possibly this delayed presentation was occasioned by her mental state (8). It is also possible that this delayed presentation is a manifestation of a lack 


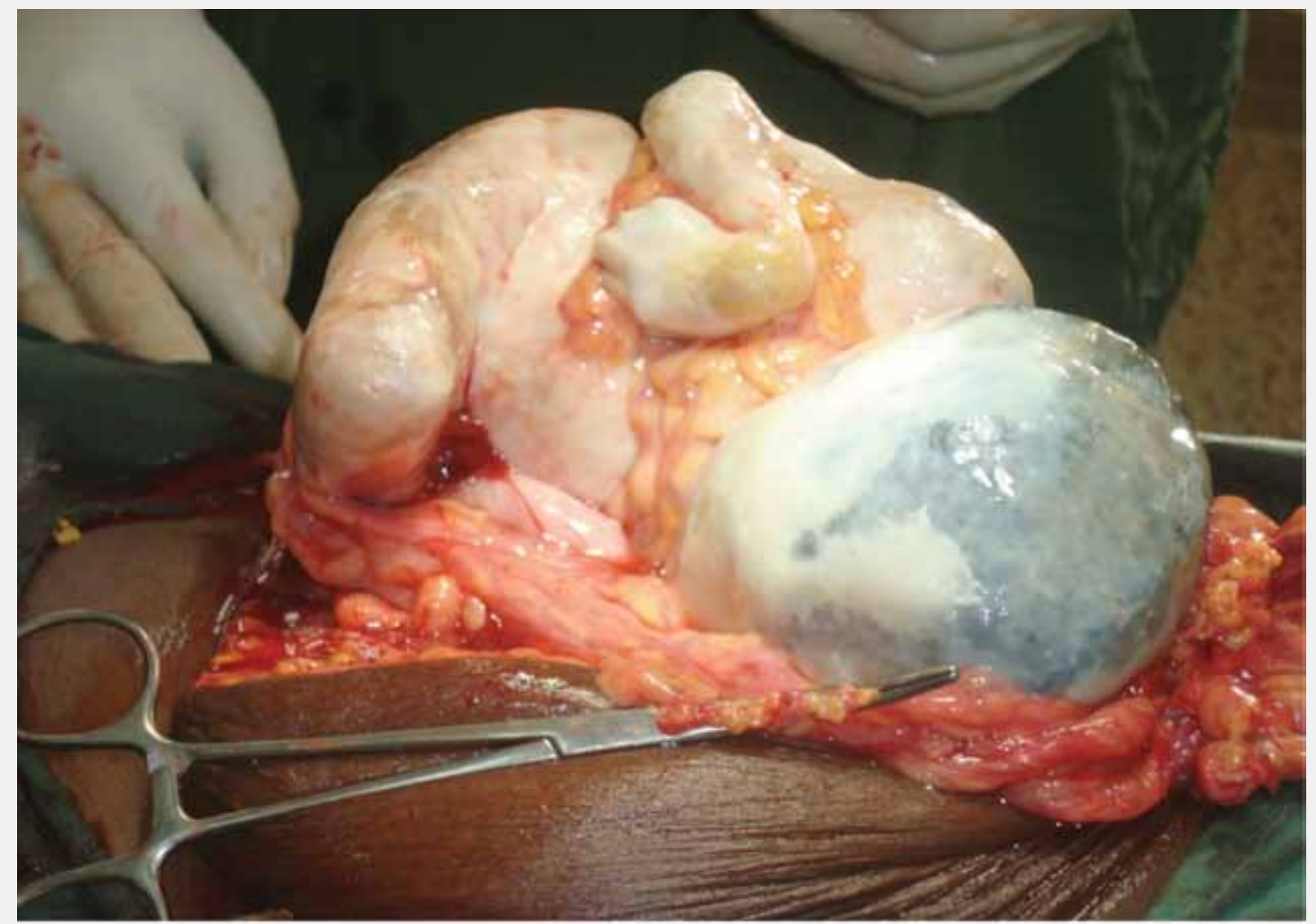

Fig. 1 Lithopedion with adherent omentum

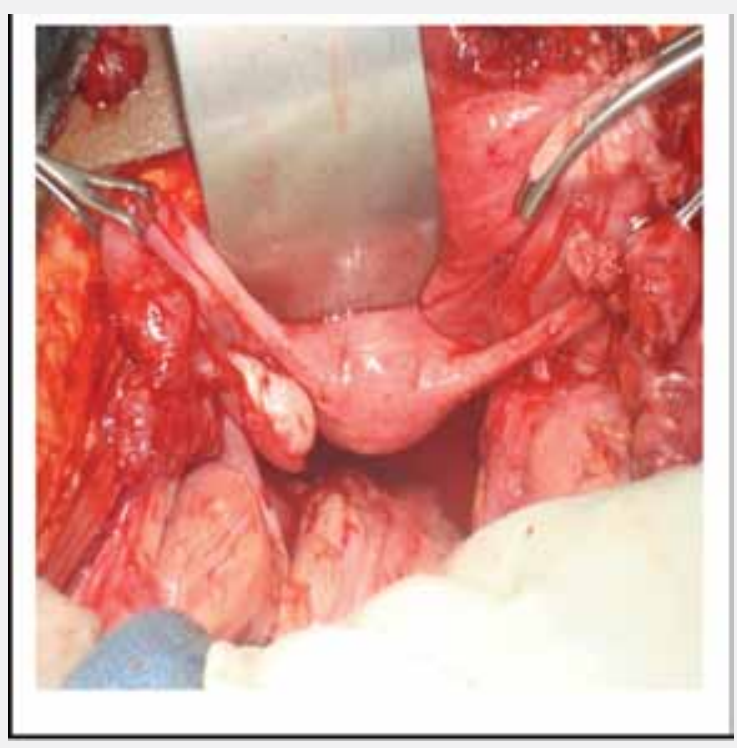

Fig. 2 Uterus, left ovary and fallopian tube visualized post operatively

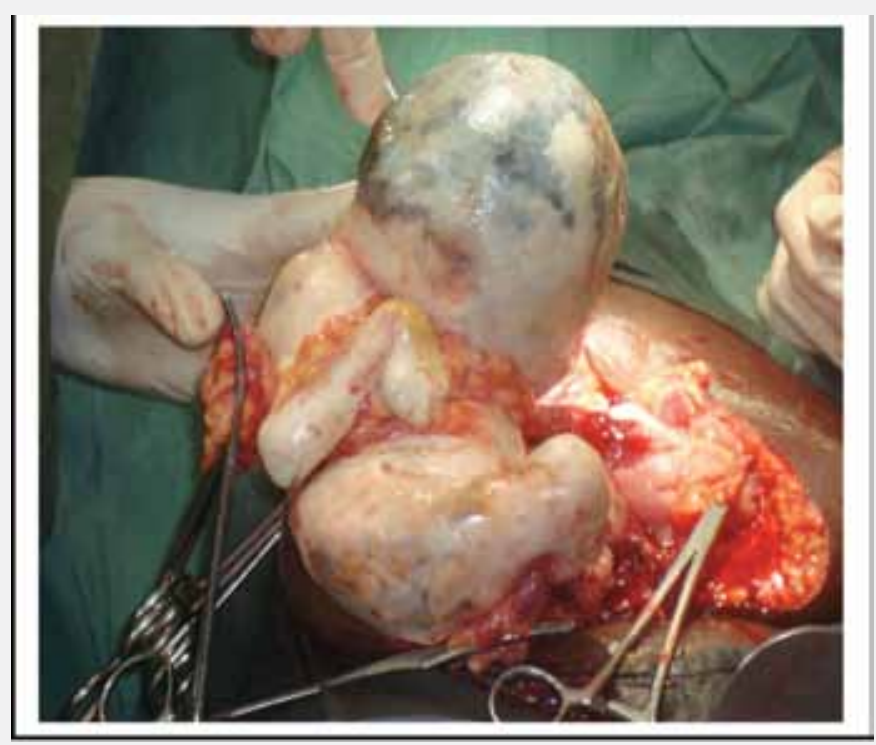

Fig. 3 The lithopedion was separated from the omentum, and removed with the adherent ovary 


\section{Lithopedion with seven years of evolution: Case Report}

Warfa K., Konya W.P.

of integration between mental health care and medical services for patients in our country. Training and provision of screening, preventive and routine medical services to mental health care providers, may unravel conditions in mental patients meriting earlier medical care $(9,10)$.

\section{Acknowledgement}

Theatre Staff of St Mary's Hospital, Nairobi.

Prof W. Stones, Dr S. Wanyoyi, Prof H. Saidi and Dr A. Murage for reading through the manuscript.

\section{Reference}

1. Zaheer SA. Acute intestinal obstruction caused by lithopaedion. Br J Surg. $1971 ; 58(5): 401-2$.

2. Bondeson J. The earliest known case of a lithopaedion. J R Soc Med. 1996;89:13-8.

3. Sun G, Li M, Lu Y. Unrecognized lithopedion with 35 years' evolution diagnosed on computed tomographic scan. Fertil Steril. 2010;94(1):341-2.
4. Jurkovic D, Mavrelos D. Catch me if you scan: ultrasound diagnosis of ectopic pregnancy. Ultrasound in Obstetrics and Gynecology. 2007;30(1):1-7.

5. Jain T, Eckert LO. Abdominal pregnancy with lithopedion formation presenting as a pelvic abscess. Obstet Gynecol. 2000 ;96(5 Pt 2):808-10.

6. Burger NZ, Hung YE, Kalof AN, et al. Lithopedion: laparoscopic diagnosis and removal. Fertil Steril. 2007 ;87(5):12089.

7. Leke RJI, Nasah BT, Shasha W, et al. Cephalopelvic disproportion at term involving a lithopedion: a case report. Int J Gynaecol Obstet. 1983;21:171-4.

8. Owens WL. Lithopedion: Report of a case with psychiatric Implications. Obstetrics \& Gynecology. 1962;19(3):401-4.

9. Druss BG, von Esenwein SA. Improving general medical care for persons with mental and addictive disorders: systematic review. Gen Hosp Psychiatry. 2006 ;28(2):145-53.

10. Morden NE, Mistler LA, Weeks WB, et al. Health care for patients with serious mental illness: Family Medicine's Role. J Am Board Fam Med. 2009;22(2):187-95. 